\title{
MACROPHTHALMOS, DISPLACEMENT BACKWARDS OF THE OPTIC NERVE ENTRANCE, WITH HISTORY OF TRAUMA AT BIRTH
}

\author{
BY \\ ERNEST THOMSON \\ STIRLING
}

Ir is a curious fact that rare cases are seldom recorded singly: one case brings out another. Although it has not yet been fully studied so close is the resemblance between the case which I am about to describe and the one put on record by MacRae in the February number of this journal that I hasten to publish it so that further references to both may be easy.

Margaret W. (Ref. No. C. 250.G.) a school child, aged 13 years, mentally slightly defective, was seen on November 15, 1928, on account of defective vision. The V.A. was recorded (by the nurse in charge) as R.E. less than 6/60, L.E. 6/12. Afterwards, when I came to examine the child, the V.A. R was no better than naming common objects quite close to the eye. Projection of light from a flash-lamp was good all over the field.

Description.-The right eye is larger than the left: roughly, the right cornea is some $2 \mathrm{~mm}$. wider than the left and the right palpebral fissure is notably wider than the left. The eye is myopic, yet there is no great error; a retinoscopy has not been done as yet, but the myopia may be taken as approximately $5 \mathrm{D}$. The left eye is hypermetropic 1D. and is in all respects normal. There is moderate right convergent strabismus: the bearing of this on the case is at present in doubt.

Continuing the description of the right eye; the anterior chamber is deep or appears so by comparison with the normal-sized fellow eye. The direct pupillary reflex to light is absent or almost absent, the consensual reflex is about normal. The tension to the finger seems to be practically the same as in the fellow eye. A large capillary naevus covers the right temple, cheek and neck regions. Ophthalmoscopically, the media are clear and the sole visible interest in the fundus so far noted lies in the optic nerve entrance. There is no disc, properly speaking. This region presents, on surface focusing, the appearance of a cavity with a dead-white background surrounded by a fringe of retinal arteries and veins. Further examination and careful focusing with parallactic displacement show that there is, as described by MacRae in his case, a goblet-like depression, the edge of which is overhanging. Over this edge the retinal arterial and venous branches dip and abruptly disappear entirely, with one exception. At the bottom of the goblet are seen a smaller and a larger vessel, indistinguishable from one another in colour, the colour being light 
red rather than red and the contour flat rather than tubular. Still deeper focusing reveals that these two vessels emerge from a funnel-shaped opening in the bottom of the goblet (to continue the simile one might almost say from the hollow stem. of the goblet) which opening is directed backwards and downwards, and pass nasalwards and upwards to disappear under the nasal overhanging edge of the goblet. With one exception all branching is invisible. The exception is a branch towards the macular region which comes off from the smaller of the two vessels in the goblet, passes under the temporal edge, and reappears as a definite artery at retinal level, thus showing that the smaller main vessel is the retinal artery and the larger, presumably, the vein. With the exception of the branch mentioned above all the branching of the main artery and vein takes place out of sight under the overhang, and one must therefore suppose that the goblet is of a very rotund shape. Further, very careful focussing reveals shadowy lines at the bottom of the goblet (not the funnel or stem) which may represent the lamina cribrosa, and also shows that a quite small upper temporal portion of the floor of the goblet is slightly pink. While, therefore on surface focusing at retinal level the background appears dead-white, on deeper focus it is greyish. The distorted shape of the opening in this case, and in MacRae's also, is in striking contrast to the normal. This distortion is hardly sufficiently shown in the drawing; the upper and lower edges of the goblet should be less curved.*

History. - So much for the appearances; next for the history given by the mother, an intelligent woman who, since she had been under anaesthetics at the time of her accouchement, knew nothing much at first hand. She states that she has had nine children and that the last four of the labours were difficult and instrumental, in the case of Margaret especially so, while another child was so injured at birth that it died. There was great swelling of the right side of Margaret's face and there was a mark above the right eye, but whether this was a bruise or the beginning of the naevus she cannot say. In any case the mark increased (so it is stated) in area until it covered the side of the face. How long it took for this to happen is impossible to ascertain with certainty. The right ear was cut, from which one may deduce with considerable certainty that a forceps blade was gripping the face on the right side. The enlargement of the right eye was noticed three days after birth. Another fact stated by the

*The picture is composite and semi-diagrammatic, and represents the mental impression obtained by the observer after focusing at various levels. It was drawn in colour by Hamblin's artist from a rough sketch and description and suffers from several inaccuracies unavoidable under the circumstances. The two main vessels in the cup are not in reality of equal width, nor does the vein taper towards the "funnel." The "funnel" itself is much too definite in outline. 
mother, and suggestive also of injury to the head, is that the child used to have what she (the mother) calls " paralytic attacks" of the left arm and leg; she has, however, been free of these for a year. There are no other naevi about the body nor any enlargement of limbs as in MacRae's case.

Although there are differences between these two cases they seem to be essentially similar. More especially there is the very remarkable coincidence of the fundus appearances with facial naevus. In the one case there is the history of possible prenatal

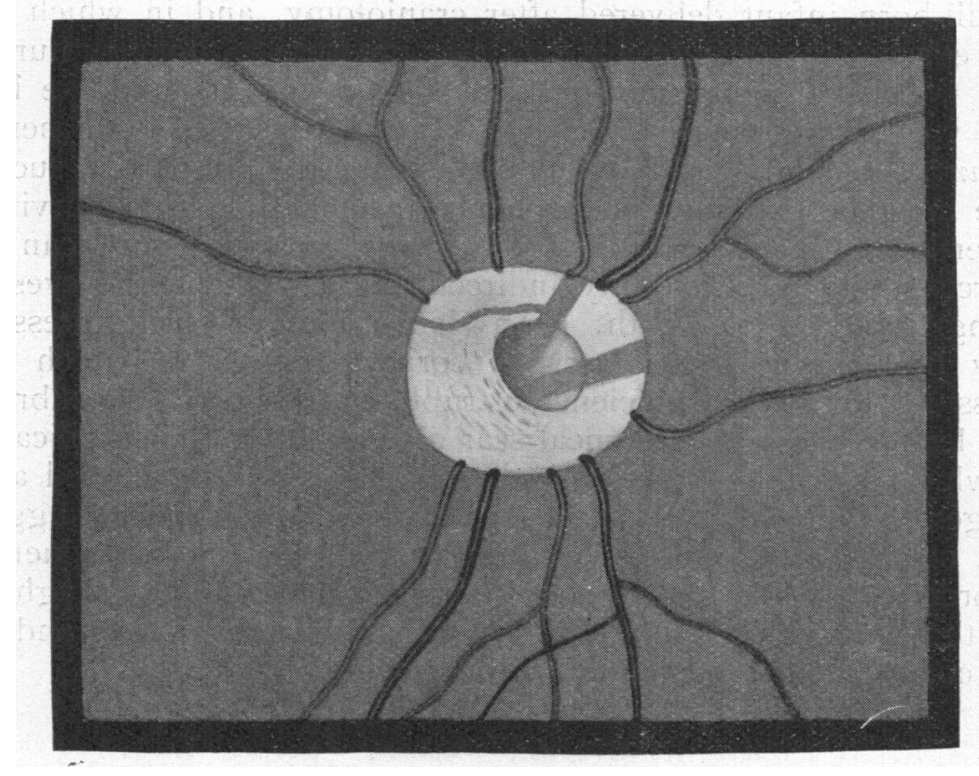

damage and in the other of damage to the eye region during birth. MacRae does not mention any enquiry into obstetric history. At the risk of being considered a crank on the subject I would ask whether birth injury may not be responsible for such cases? That birth injuries to the eye, either directly, or indirectly from skull pressure, are not yet fully appreciated is the firm opinion of Leslie Buchanan and myself, after our joint experiences published in various British and American journals since 1903. We know that a crushed head may lead to extrusion, partial or complete, of the eyeball. Is it not possible that, in cases such as these and some others labelled congenital anomalies, the eyeball has been partially dislocated, the optic nerve stretched and pulled out of its normal position and its fibres damaged, leaving the retinal vessels undamaged but stretched over the edge of the cavity left by the dislocated nerve? 
With regard to the macrophthalmos, I had discussed this with Buchanan before knowing of MacRae's case, and we then considered it possible that the eye had been stretched at birth, not progressively from within as in buphthalmos, but, since the enlarged eye was noted by the mother three days after birth, by sudden external pressure. In any case the eye does not seem to fall into the buphthalmic class.

One may say with considerable assurance that every kind of disorganisation of the eyeball is possible from pressure. Those who have studied the literature may remember that in the case of a still-born infant delivered after craniotomy, and in which the left eye had been subjected to great pressure without rupturing (the right eye being ruptured), Buchanan and I found the lens and vitreous retroverted, the lens lying against the optic nerve. (Trans. Ophthal. Soc. U.K. 1903.) While the pressure in such a case must be extreme and probably incompatible with survival, nevertheless, it is undoubted that very great pressure can be exercised, in all probability indirectly as a rule, on the eyes of living children at birth. An indication of such pressure may be seen in those cases of corneal trauma in which the pressure has been sufficient to rupture Descemet's membrane and lead to the typical corneal scar of linear type the significance of which is so often missed and which is apt to be classed as a congenital anomaly. This opportunity may be taken to suggest that ocular birth injuries in general would be more frequently recorded and better understood if obstetricians could be brought to see that accidents of this kind are generally unavoidable and do not constitute any reflection on themselves.

\section{THIRTEENTH INTERNATIONAL OPHTHAL- MOLOGICAL CONGRESS}

September 5-13, I929

\section{Under the Patronage of H.M. THE QUEEN DOWAGER OF THE NETHERLANDS}

Honorary Committee. Honorary Presidents: H.E. The Prime Minister; H.E. The Minister of Education, Art and Science; H.E. The Minister for Foreign Affairs; H.E. The Minister of the Colonies; H.E. The Minister of Labour, Trade and Industries; H.E. The Minister of Public Works; H.E. The Governor-General of the Dutch East Indies; 'The High Commissioners of H.M. The Queen in North and South Holland; The 